LETTER TO THE EDITOR

\title{
Islet xenotransplantation clinical trial: does histology show islet cells?
}

\author{
Anastasio Salazar ${ }^{1}$ and James R Wright $\mathrm{Jr}^{2}$ \\ Departments of ${ }^{1}$ Surgery (Division of Transplantation) and ${ }^{2}$ Pathology \& Laboratory Medicine (Calgary Laboratory Services), University of Calgary \\ Faculty of Medicine, Diagnostic \& Scientific Centre; 9,3535 Research Road NW, Calgary, AB, Canada T2N 2T9 \\ (Correspondence should be addressed to J R Wright Jr; Email: jim.wright@CLS.ab.ca.)
}

European Journal of Endocrinology 154 917-918

We read the paper by Valdes-Gonzalez et al. (1) with great interest. It presents the outcome of research that started with controversy focused on ethical concerns, safety issues, and the paucity of suitable preclinical data $(2-5)$. Our rodent studies on co-encapsulation of sertoli cells with islet xenografts $(6,7)$ support the scientific basis of their work but fall far short of success in non-human primates - the gold standard required by the international islet transplantation community. The clinical results presented in the paper suggest that some of the transplanted pig islets were functioning. However, the post-transplant laboratory findings are incongruous, in particular, the absence of demonstrable porcine $\mathrm{C}$-peptide in any of the islet recipients.

Perhaps the most important findings reported in this study are the histological results. According to the authors, at 'three years post transplant, one of four devices was removed from four patients, and the presence of insulin-positive cells in the transplant was demonstrated by immunohistochemistry in all four patients'. Furthermore, staining for glucagon-positive cells was reported in three of these patients. However, when we examined the photomicrographs in figures 4A (insulin-containing cells) and 4E (glucagon-containing cells), we found the immunostaining unconvincing. The cells in both $4 \mathrm{~A}$ and $4 \mathrm{E}$ have golden brown staining rather than the darker brown staining (typical of the diaminobenzidine-based immunoperoxidase method used) seen in figures 4B, 4C, and $4 \mathrm{D}$, which clearly show immunostained lymphocytic infiltrates. The cells in 4A, especially when the color images in the PDF file are slightly magnified, show variably sized course granules that look like hemosiderin, an iron-containing golden brown breakdown pigment of hemoglobin that is typically found within macrophages in areas of old hemorrhage.

We e-mailed the PDF file containing Valdes-Gonzalez et al.'s paper to four transplant pathologists at our institution and asked each one independently to review figures $4 \mathrm{~A}$ and $4 \mathrm{E}$ and offer their thoughts. All expressed similar concerns to our own and could not be certain that these figures actually represent islet cells. In other words, the few scattered 'insulin-positive' and 'glucagon-positive' cells, may be hemosiderin-laden macrophages. Cytologically, they do not strongly resemble typical islet cells. In fact, the authors themselves note that the overall architecture of the islets was to a large extent destroyed, with insulin-positive cells ... distributed throughout the collagen matrix'. Whether these cells are true islet cells can be determined by performing an iron stain for hemosiderin, a CD68 stain for macrophages, and insulin and glucagon stains with appropriate controls. In the methods section of the paper, insufficient detail is provided to critically judge the staining technique used by these authors. In particular, there is no mention of the specific antibodies, dilutions, and controls. For example, were irrelevant antibody and omit-primary antibody controls used? Did they used hydrogen peroxide to inactivate the endogenous peroxidase present in granulocytes, and was this effective?

We would recommend that the authors provide additional staining details and have the slides independently reviewed by one or more pathologists not associated with the research, but who are known within the islet transplantation community. To address this issue definitively, we believe that the authors also need to provide the paraffin blocks used so that additional sections can be cut and additional histochemical and immunohistochemical staining performed by the reviewing pathologist(s). This clinical study has landmark potential and will undoubtedly raise the hopes of diabetic patients world-wide. Therefore, it is critical to confirm whether or not viable islet cells were present at three years post transplantation, as reported.

\section{References}

1 Valdes-Gonzales R, Dorantes LM, Garibay GN, Bracho-Blanchet E, Mendez AJ, Davila-Perez R, Elliott RB, Teran L \& White DJG. 
Xenotransplantation of porcine neonatal islets of Langerhans and sertoli cells: a 4-year study. European Journal of Endocrinology $2005153419-427$.

2 Check E. Diabetes trial stirs debate on safety of xenotransplants. Nature 20024195.

3 Valdes-Gonzales R. Xenotransplantation's benefits outweigh risks. Nature $2002 \mathbf{4 2 0} 268$.

4 McKenzie IF, d'Apice AJ \& Cooper DK. Xenotransplantation trials. Lancet $20023592280-2281$

5 Birmingham K. Skepticism surrounds diabetes xenograft experiment. Nature Medicine 200281047.
6 Yang H \& Wright JR Jr. Co-encapsulation of sertoli enriched testicular cell fractions further prolongs fish-to-mouse islet xenograft survival. Transplantation $199967815-820$.

7 Yang H, Al-Jazaeri A \& Wright JR Jr. The immunoprotective effect of sertoli cells co-encapsulated with islet xenografts is not dependent upon Fas-ligand expression. Cell Transplantation 200211 799-801.

Received 26 January 2006

Accepted 7 February 2006 